Original article

\title{
First episode psychosis and comorbid ADHD, autism and intellectual disability
}

\author{
Pontus Strålin*, Jerker Hetta \\ Department of Clinical Neuroscience, Karolinska Institute, Stockholm, Sweden
}

\section{A R T I C L E I N F O}

\section{Article history:}

Received 4 July 2018

Received in revised form 24 September 2018

Accepted 24 September 2018

Available online 29 October 2018

\section{Keywords:}

Psychoses

Schizophrenia

ADHD

Autism

Mental retardation

\begin{abstract}
A B S T R A C T
Background: Comorbidity between neurodevelopmental disorders and psychotic disorders is common, but little is known about how neurodevelopmental disorders influence the presentation and outcome of first episode psychosis.

Methods: A nation-wide cohort $(\mathrm{n}=2091)$ with a first hospitalization for psychosis between 2007-2011 and at ages between 16-25 at intake was identified from Swedish population registries. Comorbid diagnoses of neurodevelopmental disorders were identified at first psychosis hospitalization and for ADHD also by dispensations of psychostimulants before the first psychosis hospitalization.

Data from the registers on hospitalizations and dispensations of antipsychotic and psychostimulant medications during the year before and 2 years after the first psychosis hospitalization were analysed. Self-harm and substance use disorders were identified by ICD10 codes at hospitalizations.

Results: $2.5 \%$ of the cohort was identified with a diagnosis of intellectual disability, $5.0 \%$ with autism and $8.1 \%$ with ADHD. A larger proportion of cases with Autism $(\mathrm{OR}=1.8, \mathrm{p}<0.05)$ and intellectual disability $(\mathrm{OR}=3.1, \mathrm{p}<0.01)$ were using antipsychotic medication year 2 compared to the rest of the cohort. Delusional disorder was more common in the autism group $(\mathrm{OR}=2.3, \mathrm{p}<0.05)$ at first psychosis hospitalization. ADHD was associated with higher risks for substance use disorders and self-harm both before and after the first psychosis hospitalization. Year 2 substance use disorder had a OR=2.6 $(\mathrm{p}<0.001)$ and self-harm OR = $4.1(\mathrm{p}<0.001)$.

Conclusions: Psychosis with comorbid ADHD is associated with high risks for substance use disorders and for self-harm, while psychosis with comorbid autism and intellectual disability is associated with longer treatment and higher doses of antipsychotic medication.
\end{abstract}

(c) 2018 Elsevier Masson SAS. All rights reserved.

\section{Introduction}

Cognitive impairments are considered to be core features of schizophrenia [1,2]. Impairments in schizophrenia have been found in cognitive domains also affected in other neurodevelopmental disorders. As in Intellectual Disability (ID) there is a generally decreased intellectual capacity on a group level in schizophrenia $[3,4]$. As in autism, impairments in theory of mind and other aspects of social cognition, impairments in sensory perception [5], and restrictions of interest [6,7] have been described. And as in ADHD, impairments of attention and executive functions $[8,9]$ have been described.

Comorbidity is common between schizophrenia and autism [7,10,11], between schizophrenia and intellectual disability [12,13], and between schizophrenia and ADHD [9,14,15].

\footnotetext{
* Corresponding author.

E-mail address: pontus.stralin@sll.se (P. Strålin).
}

Evidence has accumulated of a large overlap in genetic risk factors for schizophrenia, autism, ADHD and ID [16,17]. Genomic studies have found that many large, rare copy number variants and multiple rare coding variants in specific genes confer risk across the disorders [16].

Shared environmental early risk factors have been observed for autism and schizophrenia. Multiple studies have shown an increased risk for both disorders by advanced paternal age, pregnancy and birth complications and migration status $[18,19]$.

In the current study we wanted to explore if and how neurodevelopmental disorders (NDD's) influence the presentation and outcome of first episode psychosis.

We also wanted to study associations between specific NDD and substance use disorders (SUD) and self-harm before and after first hospital treated psychosis for the different NDD's. Based on the known high incidence of self-harm and SUD in ADHD [20,21], a hypotheses was that cases with a comorbid ADHD would have a higher probability of self-harm and SUD in conjunction with first episode psychosis (FEP). 
In order to study these issues, the Swedish population registries of inpatient care and of dispensations of prescribed drugs were used to identify and describe a cohort of young cases with first episode hospital treated psychosis and cases with comorbid NDD's.

\section{Methods}

\subsection{Data sources for the study and cohort selection}

The unique personal identity number assigned to each permanent resident in Sweden was used to link data from different registers.

The In-patient Care Diagnoses Database includes all individuals in Sweden admitted to psychiatric or general hospitals [22,23]. It includes dates for admission and discharge and ICD-10 diagnoses for every inpatient care episode in Sweden since 1987.

The study cohort was selected from the In-patient Care Diagnoses Database based on data on all hospitalizations from 1987 for the Swedish population in the selected age group. All individuals with a first occurrence of a hospitalization with a psychosis diagnosis in Sweden (as defined by the International Classification of Disease, ICD-10, F20-29) with admission after 1 July 2007 and discharge before 31 December 2011 and between age 16 and 25 at first admission were selected. 2133 cases were identified by these criteria. 42 cases had deceased before the end of 2 years after the first discharge from psychosis hospitalization, The 2091 cases alive 2 years after first discharge from psychosis hospitalization were included in the cohort. Deceased cases were identified by the Causes of Death Database, which comprises information on all deaths of Swedish residents [24]. 9 of the dead cases had a NDD diagnosis. 32 cases died by suicide, of which 5 had an NDD.

Data on hospitalizations for the cohort were collected for further analyses as described below.

Data were collected from the Swedish Prescribed Drug Database. It comprises information on all dispensations of prescribed medicines in Sweden, including the Anatomical Therapeutic Chemical code (ATC) of the dispensed substances, amount, formulation and date of prescribing and dispensing since july 2005 [25]. However, it does not cover drugs administered at hospitals.

Amounts of dispensed medication for each dispensation are reported in the register (and used in the study) as the number of "defined daily doses" (DDD) according to the WHO ATC/DDD Index [26]. Definitions of one DDD for some common medications are: clozapine $300 \mathrm{mg}$, olanzapine $10 \mathrm{mg}$, risperidone $5 \mathrm{mg}$ ( $2.7 \mathrm{mg}$ for depot), aripiprazole $15 \mathrm{mg}$, quetiapine $400 \mathrm{mg}$, paliperidone $6 \mathrm{mg}$ (2.5 for depot), methylphenidate $30 \mathrm{mg}$ and atomoxetine $80 \mathrm{mg}$. The numbers of DDD from all dispensations of any antipsychotic medication in the year before or in 2 years after the first psychosis hospitalization were summed to get yearly values of amounts of antipsychotics. The same principle of summation was used for psychostimulant medication.

For analyses of antipsychotics, ATC code N05 A was used, with the exception of N05AN (Lithium) used for bipolar disorder, and N05AA02 (levomepromazine), N05AD03 (melperone), and N05AF03 (chlorprothixene) mostly used as sedatives and as anxiolytics. For analyses of psychostimulants, the ATC code N06BA was used.

\subsection{Study design}

Data for episodes of hospitalizations and dispensations of antipsychotic and psychostimulant medications during the year before and the 2 years after the first hospitalization for psychosis were collected from the registers and analysed. Episodes of inpatient care were combined if the time between episodes in the National Patient Register was 7 days or less.
Comorbid diagnoses of ICD10 neurodevelopmental disorders at first psychosis hospitalization were used to identify cases with neurodevelopmental disorders. For autism F84, for ADHD F90 and for intellectual disability F7. Cases with any dispensations of psychostimulants in the 2 years before the FEP hospitalization were also included in the ADHD group. Cases with NDD diagnoses at earlier or later hospitalizations, but not an indexed comorbid NDD diagnosis at the first hospitalization with a psychosis diagnosis were not included since the hospitalizations before or after FEP may have strong unspecific confounding effects. For comparison cases with a Schizophrenia diagnosis (ICD10 F20) at the first hospitalization for psychosis were also identified and reported in the tables. Cases with both an identified schizophrenia diagnosis and NDD diagnosis were included in both groups in a similar way to cases with two NDD's.

To identify cases with suicide attempts and other serious selfharm, ICD10 codes X60-X84 and Y10-Y34 were detected at any type of hospitalization in the specified years.

Substance use disorders were identified by ICD-10 F1 diagnoses at hospitalizations.

\subsection{Statistical analysis}

Descriptive data for each diagnostic group were compiled on all cases identified in the groups as described above. Individuals identified in several groups were included in several compilations. Likewise, odds ratios and significance levels were calculated for all cases in a diagnostic group in relation to all other cases in the cohort, including the cases in the other diagnostic groups under study.

Significance levels for Odds Ratios were calculated based on Wald test statistics and for quantitative measures with the Wilcoxon method.

Descriptive analyses and odds ratio analyses were made with the $\mathrm{R}$ software [27].

A multivariate logistic regression was made on associations between self-harm and SUD and ADHD in year 2 after the first psychosis hospitalization, initially including an interaction factor. An optimal model was found by AIC (Akaike Information Criterion) guided model exploration.

The study was approved by the Regional Ethical Review Board in Stockholm, Sweden (ref 2014/481-31/4).

\section{Results}

\subsection{Frequencies of Autism, ADHD and Intellectual disability in the cohort}

53 cases were identified with intellectual disability (2.5\% of the cohort), of which 37 had mild (F70), 5 moderate (F71) and 11 unspecified (F79) types. 105 cases were identified with autism (5.0\% of the cohort), of which 76 had Asperger's syndrome (F845), 13 had autistic disorder (F841), 7 had atypical autism (F(840), and 10 had unspecified pervasive developmental disorder (F849). 170 cases were identified with ADHD (8.1\% of the cohort), of which 93 had a comorbid ICD10 diagnosis of ADHD (F90) at first psychosis hospitalization.

Some cases were identified with more than one NDD. 24 cases had both autism and ADHD, 9 cases had autism and ID, 6 cases had ID and ADHD and no case had all three.

\subsection{Characteristics of the neurodevelopmental subpopulations before and at first psychosis hospitalization}

Median age at first psychosis hospitalization was slightly lower for the cases with neurodevelopmental diagnoses compared to the 
rest of the cohort (Table 1a). Gender distribution was not significantly different in the groups with autism or ID compared to the rest of the cohort. For ADHD there was a significantly higher proportion of male cases compared to the rest of the cohort (Table 1a). The median duration of the first psychosis hospitalization was longer in the Autism group as compared to the rest of the cohort, but not as long as for cases with a Schizophrenia diagnosis at first hospitalization. The median durations were slightly shorter for the Intellectual disability and ADHD groups. Cases identified with both ADHD and autism had longer duration of their first psychosis hospitalizations, the median duration was 37 days.

The proportion of cases with a diagnosis of schizophrenia (F20), Brief psychosis (F23), Schizoaffective disorder (F25) or Unspecified psychosis (F29) at the first psychosis hospitalization were similar in the subgroups with comorbid neurodevelopmental diagnoses as compared to the whole cohort (Table 1a). Delusional disorder (F22) was significantly more common in the Autism group compared to the rest of the cohort with an Odds Ratio of 2.3 (95\% CI: 1.1-4.3).

Hospitalizations for SUD (ICD10 F1) in the year before the first psychosis hospitalization were significantly more common in the ADHD group (Table $1 \mathrm{~b}$ ). In a similar pattern a comorbid diagnosis of SUD was significantly more common in the ADHD group at the first psychosis hospitalization (Table 1a). Self-harm was significantly more common in the ADHD group in the year before the first psychosis hospitalization.

Prescriptions of antipsychotic and psychostimulant medication were measured the year before first psychosis intake. A majority of cases with autism or ID had dispensations of antipsychotics before the first psychosis hospitalization and a significantly higher proportion than in the full cohort (Table 1b). Among cases with ADHD dispensations of antipsychotics was also significantly more common.

\subsection{Outcome of the neurodevelopmental subpopulations in the 2 years after the first psychosis hospitalization}

$32 \%$ of the cases in the full cohort had any psychosis hospitalization in the first year and $23 \%$ in year 2 (Table 1c). $62 \%$ had any dispensation of antipsychotic medication year 2, down from $80 \%$ in the first year after discharge. Altogether $65 \%$ had either any dispensation of antipsychotics or psychosis hospitalization the second year. For the ID and Autism groups significantly higher proportions of cases had dispensations of antipsychotics in the first two years. Year 2 ORs were 3.1 for ID (95\% CI: 1.6-6.8) and 1.8 for autism (95\% CI: 1.1-2.8) (Table 1c). For the ADHD group, the proportions with antipsychotic dispensations were similar to the cohort as a whole. The proportions of NDD cases with psychosis hospitalizations were not significantly different from the rest of the cohort. 11 of the 24 cases (46\%) identified with both ADHD and autism had psychosis hospitalizations the second year, giving an OR of 2.9, $\mathrm{p}<0.05$ compared to the rest of the cohort.

The amounts of antipsychotics were measured as DDD dispensed per year. The amounts were significantly higher in the ID and Autism groups both the first and the second years

Table 1

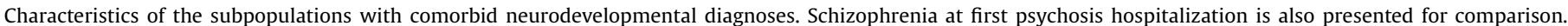

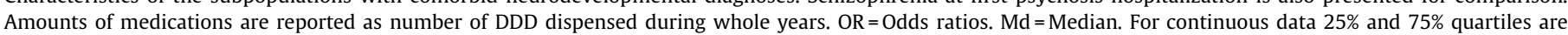
presented in (). P-values are indicated by ${ }^{*}$ for $\mathrm{p}$-value $<0.05,{ }^{* *}<0.01$ and ${ }^{* * *}<0.001$.

a. The first hospitalization for psychosis. Cases are only counted for one Psychosis diagnosis but may be counted independently for comorbid substance use disorder and self-harm diagnoses.

\begin{tabular}{|c|c|c|c|c|c|}
\hline & $\begin{array}{l}\text { Cohort } \\
n=2091\end{array}$ & $\begin{array}{l}\text { Intellectual disability (F7) } \\
n=53\end{array}$ & $\begin{array}{l}\text { Autism (F84) } \\
\mathrm{n}=105\end{array}$ & $\begin{array}{l}\text { ADHD (F90) } \\
\mathrm{n}=170\end{array}$ & $\begin{array}{l}\text { Schizophrenia (F20) } \\
n=229\end{array}$ \\
\hline Proportion Male & $64 \%(n=1336)$ & $60 \%(n=32)$ OR $=0.86$ & $69 \%(n=72) O R=1.25$ & $68 \%(n=116) O R=1.23$ & $74 \%(n=169)$ OR $=1.68^{* *}$ \\
\hline Median age at intake & $\mathrm{Md}=21.2,(19.2,23.1)$ & $\mathrm{Md}=20.6,(18.3,22.8)$ & $\mathrm{Md}=20.6,(18.2,22.4)^{* *}$ & $\mathrm{Md}=20.3,(18.5,22.5)^{* * *}$ & $\mathrm{Md}=21.4,(19.3,23.4)$ \\
\hline Median days in hospital & $\mathrm{Md}=18,(7,41)$ & $\mathrm{Md}=20,(7,40)$ & $\mathrm{Md}=26,(10,87)^{* *}$ & $\mathrm{Md}=11.5,(4.2,37.5)^{*}$ & $\mathrm{Md}=41,(14,90)^{* * *}$ \\
\hline Substance use disorder & $18 \%(n=380)$ & $8 \%(n=4)$ OR $=0.36$ & $8 \%(n=8) O R=0.36^{* *}$ & $24 \%(n=41)$ OR $=1.48^{*}$ & $16 \%(n=37) O R=0.85$ \\
\hline Self-harm & $4 \%(n=83)$ & $\mathrm{n}<4$ & $8 \%(n=8) O R=2.1$ & $6 \%(n=10)$ OR $=1.58$ & $3 \%(n=6)$ OR $=0.62$ \\
\hline Schizophrenia (F20) & $11 \%(n=229)$ & $11 \%(n=6) O R=1.04$ & $8 \%(n=8)$ OR $=0.66$ & $11 \%(n=18)$ OR $=0.96$ & \\
\hline Schizoaffective (F25) & $4 \%(n=88)$ & $\mathrm{n}<4$ & $6 \%(n=6) O R=1.41$ & $4 \%(n=6)$ OR $=0.82$ & \\
\hline Delusional disorder (F22) & $5 \%(n=107)$ & $\mathrm{n}<4$ & $10 \%(n=11)$ OR $=2.3^{*}$ & $4 \%(n=7)$ OR $=0.78$ & \\
\hline Brief psychosis (F23) & $35 \%(n=742)$ & $30 \%(n=16) O R=0.78$ & $28 \%(n=29)$ OR $=0.68$ & $39 \%(n=67)$ OR $=1.2$ & \\
\hline Psychosis NOS (F29) & $42 \%(n=882)$ & $49 \%(n=26)$ OR $=1.33$ & $47 \%(n=49)$ OR $=1.21$ & $39 \%(n=67)$ OR $=0.88$ & \\
\hline
\end{tabular}

b. Characteristics for the year before the first psychosis hospitalization.

\begin{tabular}{|c|c|c|c|c|c|}
\hline & $\begin{array}{l}\text { Cohort } \\
n=2091\end{array}$ & $\begin{array}{l}\text { Intellectual disability (F7) } \\
\mathrm{n}=53\end{array}$ & $\begin{array}{l}\text { Autism (F84) } \\
\mathrm{n}=105\end{array}$ & $\begin{array}{l}\text { ADHD (F90) } \\
\mathrm{n}=170\end{array}$ & $\begin{array}{l}\text { Schizophrenia (F20) } \\
\mathrm{n}=229\end{array}$ \\
\hline Substance use disorders & $11 \%(n=231)$ & $\mathrm{n}<4$ & $7 \%(\mathrm{n}=7) \mathrm{OR}=0.56$ & $25 \%(\mathrm{n}=43) \mathrm{OR}=3.12^{* * *}$ & $12 \%(n=27)$ OR $=1.09$ \\
\hline Self-harm & $4 \%(n=88)$ & $\mathrm{n}<4$ & $7 \%(n=7)$ OR $=1.68$ & $9 \%(n=16)$ OR $=2.67^{* * *}$ & $5 \%(\mathrm{n}=12)$ OR $=1.3$ \\
\hline Antipsychotics & $32 \%(n=678)$ & $62 \%(\mathrm{n}=33) \mathrm{OR}=3.56^{* * *}$ & $60 \%(\mathrm{n}=63) \mathrm{OR}=3.34^{* * *}$ & $49 \%(n=83)$ OR $=2.13^{* * *}$ & $50 \%(\mathrm{n}=114)$ OR $=2.28^{* * *}$ \\
\hline DDD antipsychotics & $\mathrm{Md}=97,(28,250)$ & $M d=133,(30,268)$ & $\mathrm{Md}=166,(53,396)^{* *}$ & $\mathrm{Md}=106,(30,278)$ & $\mathrm{Md}=157,(57,340)^{* * *}$ \\
\hline
\end{tabular}

c. Characteristics for the 2 year after discharge from the first psychosis hospitalization.

\begin{tabular}{|c|c|c|c|c|c|}
\hline & $\begin{array}{l}\text { Cohort } \\
n=2091\end{array}$ & $\begin{array}{l}\text { Intellectual disability (F7) } \\
\mathrm{n}=53\end{array}$ & $\begin{array}{l}\text { Autism (F84) } \\
\mathrm{n}=105\end{array}$ & $\begin{array}{l}\text { ADHD (F90) } \\
\mathrm{n}=170\end{array}$ & $\begin{array}{l}\text { Schizophrenia (F20) } \\
\mathrm{n}=229\end{array}$ \\
\hline Substance use disorder year 1 & $15 \%(n=305)$ & $9 \%(n=5) O R=0.6$ & $6 \%(n=6) O R=0.34^{*}$ & $25 \%(\mathrm{n}=42) \mathrm{OR}=2.07^{* * *}$ & $13 \%(n=30)$ OR $=0.87$ \\
\hline Substance use disorder year 2 & $11 \%(n=235)$ & $\mathrm{n}<4$ & $\mathrm{n}<4$ & $23 \%(\mathrm{n}=39)$ OR $=2.62^{* * *}$ & $6 \%(n=14)$ OR $=0.48^{*}$ \\
\hline Self-harm year 1 & $6 \%(n=130)$ & $\mathrm{n}<4$ & $7 \%(n=7)$ OR $=1.08$ & $14 \%(n=23)$ OR $=2.65^{* * *}$ & $5 \%(n=11)$ OR $=0.74$ \\
\hline Self-harm year 2 & $4 \%(n=76)$ & $8 \%(n=4)$ OR $=2.23$ & $6 \%(n=6)$ OR $=1.66$ & $11 \%(n=19)$ OR $=4.11^{* * *}$ & $4 \%(n=9)$ OR $=1.1$ \\
\hline Psychosis hospitalization year 1 & $32 \%(n=673)$ & $32 \%(n=17)$ OR $=0.99$ & $37 \%(n=39)$ OR $=1.26$ & $34 \%(n=58)$ OR $=1.1$ & $46 \%(\mathrm{n}=106) \mathrm{OR}=1.97^{* * *}$ \\
\hline Psychosis hospitalization year 2 & $23 \%(n=481)$ & $28 \%(n=15)$ OR $=1.33$ & $29 \%(n=30)$ OR $=1.36$ & $23 \%(n=39)$ OR $=1$ & $31 \%(\mathrm{n}=70)$ OR $=1.55^{* *}$ \\
\hline Antipsychotics year 1 & $80 \%(n=1674)$ & $98 \%(n=52)$ OR $=13.34^{*}$ & $89 \%(n=93)$ OR $=1.99 *$ & $81 \%(n=138)$ OR $=1.08$ & $87 \%(\mathrm{n}=199)$ OR $=1.74^{* *}$ \\
\hline Antipsychotics year 2 & $62 \%(n=1289)$ & $83 \%(n=44)$ OR $=3.11^{* *}$ & $73 \%(n=77)$ OR $=1.76^{*}$ & $67 \%(n=114)$ OR $=1.29$ & $75 \%(n=172)$ OR $=2.01^{* * *}$ \\
\hline DDD antipsychotics year 1 & $\mathrm{Md}=216,(93,420)$ & $M d=388,(196,672)^{* * *}$ & $M d=359,(155,654)^{* * *}$ & $M d=264,(98,627)^{* *}$ & $M d=339,(177,586)^{* * *}$ \\
\hline DDD antipsychotics year 2 & $\mathrm{Md}=252,(108,478)$ & $\mathrm{Md}=402,(212,590)^{* *}$ & $\mathrm{Md}=404,(168,652)^{* * *}$ & $\mathrm{Md}=308,(117,619)^{*}$ & $\mathrm{Md}=348,(243,627)^{* * *}$ \\
\hline
\end{tabular}


compared to the rest of the cohort and even higher than for the schizophrenia group (Table 1c). Median doses in all groups were significantly higher after compared to before the first psychosis hospitalization (Table $1 \mathrm{~b}$ and $\mathrm{c}$ ).

Dispensations of psychostimulants in the ADHD group were less common year two after (69 cases, $41 \%$ ), compared to the year before first psychosis hospitalization (113 cases, 66\%). The median amounts of psychostimulants was higher year 2 (336 DDD) compared to the year before the first psychosis hospitalization (198 DDD). 52 of the 69 cases (75\%) using psychostimulants year 2 also had antipsychotic medication. 57 cases had dispensations of methylphenidate, 17 cases had dispensations of atomoxetine. 4 cases had dispensations of other types of psychostimulants.

Hospitalizations for SUD after discharge were significantly more common in the ADHD group with $23 \%$ of the ADHD cases compared to $11 \%$ in the whole cohort year 2, giving an OR of 2.6. The first year the numbers were $25 \%$ in the ADHD group, compared to $15 \%$ in the whole cohort (Table 1c). Hospitalizations for self-harm were significantly more common in the ADHD group, with a proportion year 2 of $11 \%$ in the ADHD group compared to $4 \%$ in the whole cohort, giving an OR of 4.1 (Table 1c). The first year the numbers were 13\% in the ADHD group compared to $6 \%$ in the whole cohort.

\subsection{Association between substance use disorders and self-harm in the cohort}

There was a significant association between any substance use disorder and any self-harm in the second year after the first psychosis hospitalization in the cohort with an OR of 4.7, $\mathrm{p}=0.002$. A multivariate analysis of the association between self-harm and substance use disorder and ADHD showed independent associations between substance use disorder and self-harm and ADHD and self-harm with multivariate OR's of $5.4, \mathrm{p}<0.001$, and 3.2 , $\mathrm{p}<0.001$.

\section{Discussion}

The prevalences presented for Autism and ID in the study are high compared to the general population, but are likely underestimations, since identification was dependent on registrations of comorbid diagnoses at the first psychosis hospitalizations.

A larger part of cases with comorbid ADHD was probably identified since both cases with registration of a comorbid ADHD diagnosis at first psychosis hospitalization and cases with previous psychostimulant treatment were included.

Concerning the comorbidity between psychotic disorders and ADHD there are some complicating aspects to consider. A large part of the ADHD cases had dispensations of psychostimulants before their first psychosis hospitalizations. Psychostimulants have been associated with psychotic symptoms as side-effects [28], and may thus have contributed to the development of the psychotic disorders in some ADHD cases. The proportion of cases in the ADHD group with psychostimulants after the FEP hospitalization was also lower compared to before. At the same time, most cases with psychostimulants after discharge from the psychosis hospitalization also had prescriptions of antipsychotics in parallel, indicating that for some patients with ADHD, the combination is considered to be of some value and reasonably tolerated.

As previously reported [20], there was a high comorbidity between ADHD and substance use disorders both before and after FEP hospitalization, Substance induced psychotic states have probably contributed more to the development of psychotic disorders in the ADHD group compared to the rest of the cohort [29].

The risk for suicide, suicide attempts and other types of selfharm are high in ADHD in general [21]. In line with that there was a highly increased risk for self-harm in cases with comorbid ADHD after a FEP, and also the year before FEP. The risk for self-harm was found to be independently increased by substance use disorders and by ADHD in the cohort. The high risk for self-harm in cases with psychosis and comorbid substance use disorders and/ or ADHD calls for careful monitoring and high accessibility for care and support in these groups after FEP.

The comorbidity between ADHD and psychotic disorder was found to be more common for men, as has recent been observed [15].

A significantly higher proportion of cases with a comorbid ID or autism and to a less extent also ADHD compared to the whole cohort had prescriptions of antipsychotics before their first psychosis hospitalization. Median amounts used were low though, compared to after the FEP hospitalizations. It is known that antipsychotic medication is used to a large extent in autism and ID for other indications than psychosis [30,31]. In the current study we do not have information on how many of the cases with antipsychotic medication before the first psychosis hospitalization were treated for psychotic symptoms and how many were treated for other symptoms such as irritability [30].

Cases with autism or ID have a higher probability of continued antipsychotic medication year 2 after the FEP hospitalization, as compared to the rest of the cohort. They also use higher median dosages and similar to levels in the group with schizophrenia. As there is no indication of a reduced risk for hospitalizations, these findings may indicate that their psychotic symptoms respond less to treatment. The high comorbidity with autism found in a clinical sample of chronic psychotic disorders may support a hypothesis of more severe psychotic disorders in cases with comorbid autism [10], though the issue needs further study to clarify.

The high comorbidity between psychotic disorders and neurodevelopmental disorders, particularly autism, has generated hypotheses regarding a psychosis vulnerability in NDD's. Information processing, including perceptual processing in NDD's is postulated to be impaired in various aspects compared to control populations and the impairments may increase the risk for transitions into psychotic states [19,32-35]. Further studies are needed to elucidate if such vulnerabilities are limited to subtypes of the neurodevelopmental disorders [36,37] or if predictors or additional risk factors for transitions into psychosis for individuals with NDD's may be found.

From the current study it is possible to speculate that self-harm and SUD may be risk factors for psychosis (or psychosis hospitalization) in cases with ADHD. And maybe irritability or milder psychotic symptoms needing antipsychotic treatment are risk factors for psychosis hospitalization in cases with autism or intellectual disability. These questions would need further studies based on cohorts of NDD cases to examine.

The finding of a higher risk for delusional disorder in autism may have a relation to a higher delusion-proneness in autism [38] and also maybe to deficits in social cognition characteristic for autism [5,39].

\section{Limitations}

Outcome is measured in terms of proportions of cases with hospitalizations and dispensations of medication in the cohort in the years after discharge from the first psychosis hospitalization. These measures have clear limitations. They only indirectly and to limited extents represent stabilization of symptoms and functional recovery. Even so, they probably reflect the severity of the psychotic and comorbid disorders to some extent on a group level. Concerning the psychotic disorder, it is likely that many cases with no dispensations of antipsychotics and no psychosis hospitalizations in the first two years have a less severe psychotic disorder 
as compared to many cases with ongoing antipsychotic medication and/or new psychosis hospitalizations.

\section{Conclusions}

Psychosis with comorbid ADHD is associated with substance use disorders and self-harm both before and after the first psychosis hospitalization, while psychosis with comorbid autism or intellectual disability is associated with longer treatment and higher doses of antipsychotic medication after the first psychosis hospitalization, as well as antipsychotic treatment before the first psychosis hospitalization. The findings point to the importance of considering comorbid NDD's in the treatment and management of first episode psychosis, although more studies are needed to further clarify the relations.

\section{Conflict of interest}

The authors have no conflict of interest to disclose.

\section{Ethical approval}

The study has been approved by the Regional Ethics Committee in Stockholm

\section{Acknowledgements}

The study was supported through grants from the Swedish Research Council.

\section{References}

[1] Gold J.M., Weinberger DR. Cognitive deficits and the neurobiology of schizophrenia. Curr Opin Neurobiol 1995;5:225-30.

[2] Green MF. Cognitive impairment and functional outcome in schizophrenia and bipolar disorder. J Clin Psychiatry 2006;67(Suppl. 9)3-8 discussion 36-42.

[3] Wells R, Swaminathan V, Sundram S, Weinberg D, Bruggemann J, Jacomb I, et al. The impact of premorbid and current intellect in schizophrenia: cognitive, symptom, and functional outcomes. NPJ Schizophr 2015;1:15043, doi:http://dx.doi.org/10.1038/npjschz.2015.43.

[4] Badcock JC, Dragović M. Waters FAV, Jablensky A. Dimensions of intelligence in schizophrenia: evidence from patients with preserved, deteriorated and compromised intellect. J Psychiatr Res 2005;39:11-9, doi:http://dx.doi.org/ 10.1016/j.jpsychires.2004.05.002.

[5] van Schalkwyk GI, Volkmar FR, Corlett PR. A predictive coding account of psychotic symptoms in autism Spectrum disorder. J Autism Dev Disord 2017;47:1323-40, doi:http://dx.doi.org/10.1007/s10803-017-3065-9.

[6] Green MF, Horan WP, Lee J. Social cognition in schizophrenia. Nat Rev Neurosci 2015;16:620-31, doi:http://dx.doi.org/10.1038/nrn4005.

[7] Kincaid DL, Doris M, Shannon C, Mulholland C. What is the prevalence of autism spectrum disorder and ASD traits in psychosis? A systematic review. Psychiatry Res 2017;250:99-105, doi:http://dx.doi.org/10.1016/j. psychres.2017.01.017.

[8] Gold JM, Robinson B, Leonard CJ, Hahn B, Chen S, McMahon RP, et al. Selective attention, working memory, and executive function as potential independent sources of cognitive dysfunction in schizophrenia. Schizophr Bull 2017, doi: http://dx.doi.org/10.1093/schbul/sbx155.

[9] Dalsgaard S, Mortensen PB, Frydenberg M, Maibing CM, Nordentoft M, Thomsen PH. Association between Attention-Deficit Hyperactivity Disorder in childhood and schizophrenia later in adulthood. Eur Psychiatry 2014;29:25963, doi:http://dx.doi.org/10.1016/j.eurpsy.2013.06.004.

[10] Hallerbäck MU, Lugnegård T, Gillberg C. Is autism spectrum disorder common in schizophrenia? Psychiatry Res 2012;198:12-7, doi:http://dx.doi.org/ 10.1016/j.psychres.2012.01.016.

[11] Maibing CF, Bøcker Pedersen C, Benros ME, Mortensen PB, Dalsgaard S, Nordentoft M. Risk of schizophrenia increases after all child and adolescent psychiatric disorders: a nationwide study. Schizophr Bull 2015;41:963-70, doi:http://dx.doi.org/10.1093/schbul/sbu119.

[12] Aman H, Naeem F, Faroog S, Ayub M. Prevalence of nonaffective psychosis in intellectually disabled clients: Systematic review and meta-analysis. Psychiatr Genet 2016;26:145-55, doi:http://dx.doi.org/10.1097/ YPG.0000000000000137.
[13] Cooper S-A, Smiley E, Morrison J, Allan L, Williamson A, Finlayson J, et al. Psychosis and adults with intellectual disabilities. Soc Psychiatry Psychiatr Epidemiol 2007;42:530-6, doi:http://dx.doi.org/10.1007/s00127-007-0197-9.

[14] Marwaha S, Thompson A, Bebbington P, Singh SP, Freeman D, Winsper C, et al. Adult attention deficit hyperactivity symptoms and psychosis: epidemiological evidence from a population survey in England. Psychiatry Res 2015;229:49-56, doi:http://dx.doi.org/10.1016/j.psychres.2015.07.075.

[15] Solberg BS, Halmøy A, Engeland A, Igland J, Haavik J, Klungsøyr K. Gender differences in psychiatric comorbidity: a population-based study of 40000 adults with attention deficit hyperactivity disorder. Acta Psychiatr Scand 2017:137:176-86, doi:http://dx.doi.org/10.1111/acps.12845.

[16] Owen MJ, O’Donovan MC. Schizophrenia and the neurodevelopmental continuum:evidence from genomics. World Psychiatry 2017;16:227-35, doi: http://dx.doi.org/10.1002/wps.20440.

[17] Smoller JW. Identification of risk loci with shared eff ects on fi ve major psychiatric disorders: a genome-wide analysis. Lancet 2013;381:1371-9, doi: http://dx.doi.org/10.1016/S0140-6736(12)62129-1.

[18] Hamlyn J, Duhig M, Mcgrath J, Scott J. Modifiable risk factors for schizophrenia and autism - shared risk factors impacting on brain development 2 . Neurobiol Dis 2013;53:3-9, doi:http://dx.doi.org/10.1016/j.nbd.2012.10.023.

[19] Chisholm K, Lin A, Abu-Akel A, Wood SJ. The association between autism and schizophrenia spectrum disorders: a review of eight alternate models of cooccurrence. Neurosci Biobehav Rev 2015;55:173-83, doi:http://dx.doi.org/ 10.1016/j.neubiorev.2015.04.012.

[20] Levy S, Katusic SK, Colligan RC, Weaver AL, Killian JM, Voigt RG, et al. Childhood ADHD and risk for substance dependence in adulthood: a longitudinal, population-based study. PLoS One 2014;9:, doi:http://dx.doi.org/10.1371/ journal.pone.0105640.

[21] Allely CS. The association of ADHD symptoms to self-harm behaviours: systematic PRISMA review. BMC Psychiatry 2014;14:, doi:http://dx.doi.org/ 10.1186/1471-244X-14-133.

[22] In-patient care diagnoses database. Stockholm, Sweden: National Board of Health and Welfare; 2017. http://www.socialstyrelsen.se/statistics/ statisticaldatabase.

[23] Ludvigsson JF, Andersson E, Ekbom A, Feychting M, Kim J-L, Reuterwall C, et al. External review and validation of the Swedish national inpatient register. BMC Public Health 2011;11:450, doi:http://dx.doi.org/10.1186/1471-2458-11-450.

[24] Causes of death database. Stockholm, Sweden: National Board of Health and Welfare; 2017. http://www.socialstyrelsen.se/statistics/statisticaldatabase.

[25] Prescribed drugs database. Stockholm, Sweden: National Board of Health and Welfare; 2017. http://www.socialstyrelsen.se/statistics/statisticaldatabase.

[26] A.T.C./D.D.D. Index. WHO Collab Cent drug stat methodol n.d. http://www. whocc.no/atc_ddd_index/.

[27] R: a language and environment for statistical computing. Vienna, Austria: R Foundation for Statistical Computing; 2016. https://www.r-project.org/.

[28] Mosholder AD, Gelperin K, Hammad TA, Phelan K, Johann-Liang $R$. Hallucinations and other psychotic symptoms associated with the use of Attention-Deficit/Hyperactivity disorder drugs in children. Pediatrics 2009;123:611-6, doi:http://dx.doi.org/10.1542/peds.2008-0185.

[29] Levy E, Traicu A, Iyer S, Malla A, Joober R. Psychotic disorders comorbid with attention-deficit hyperactivity disorder: an important knowledge gap. Can J Psychiatry 2015;60:S48-52.

[30] Madden JM, Lakoma MD, Lynch FL, Rusinak D, Owen-Smith AA, Coleman KJ, et al. Psychotropic medication use among insured children with autism Spectrum disorder. J Autism Dev Disord 2017;47:144-54, doi:http://dx.doi. org/10.1007/s10803-016-2946-7.

[31] Stepanova E, Dowling S, Phelps M, Findling RL. Pharmacotherapy of emotional and behavioral symptoms associated with autism spectrum disorder in children and adolescents. Dialogues Clin Neurosci 2017;19:395-402.

[32] Robertson CE, Baron-Cohen S. Sensory perception in autism. Nat Rev Neurosci 2017;18:671-84, doi:http://dx.doi.org/10.1038/nrn.2017.112.

[33] Marco EJ, Hinkley LBN, Hill SS, Nagarajan SS. Sensory processing in autism: a review of neurophysiologic findings. Pediatr Res 2011;69:48R-54R, doi:http:// dx.doi.org/10.1203/PDR.0b013e3182130c54.

[34] Tavassoli T, Miller LJ, Schoen SA, Nielsen DM, Baron-Cohen S. Sensory overresponsivity in adults with autism spectrum conditions. Autism 2014, doi: http://dx.doi.org/10.1177/1362361313477246.

[35] Sinha P, Kjelgaard MM, Gandhi TK, Tsourides K, Cardinaux AL, Pantazis D, et al. Autism as a disorder of prediction. Proc Natl Acad Sci U S A 2014;111:15220-5, doi:http://dx.doi.org/10.1073/pnas.1416797111.

[36] Lai M-C, Lombardo MV, Baron-Cohen S. Autism. Lancet 2014;383:896-910, doi:http://dx.doi.org/10.1016/S0140-6736(13)61539-1.

[37] Faraone SV, Asherson P, Banaschewski T, Biederman J, Buitelaar JK, RamosQuiroga JA, et al. Attention-deficit/hyperactivity disorder. Nat Rev Dis Prim 2015;1:15020, doi:http://dx.doi.org/10.1038/nrdp.2015.20.

[38] Louzolo A, Gustavsson P, Tigerström L, Ingvar M, Olsson A, Petrovic P. Delusionproneness displays comorbidity with traits of autistic-spectrum disorders and ADHD. PLoS One 2017;12:, doi:http://dx.doi.org/10.1371/journal. pone.0177820.

[39] Baron-Cohen S, Leslie AM, Frith U. Does the autistic child have a "theory of mind"? Cognition 1985;21:37-46, doi:http://dx.doi.org/10.1016/0010-0277 (85)90022-8. 